\title{
A new compact solid-state neutral particle analyser at ASDEX Upgrade: Setup and physics modeling
}

P. A. Schneider, ${ }^{1}$ H. Blank, ${ }^{1}$ B. Geiger, ${ }^{1}$ K. Mank ${ }^{1}$ S. Martinov,${ }^{1}$ F. Ryter, ${ }^{1}$ M. Weiland, ${ }^{1}$ A. Weller, ${ }^{1}$ and the ASDEX Upgrade Team ${ }^{\mathrm{a}) 1}$

Max-Planck-Institut für Plasmaphysik, Garching, Germany

At ASDEX Upgrade (AUG) a new compact solid-state detector has been installed to measure the energy spectrum of fast neutrals based on the principle described by Shinohara et. al. ${ }^{1}$. The diagnostic relies on the usual charge exchange of supra thermal fast-ions with neutrals in the plasma. Therefore, the measured energy spectra directly corresponds to that of confined fast-ions with a pitch angle defined by the line of sight of the detector.

Experiments in AUG showed the good signal to noise characteristics of the detector. It is energy calibrated and can measure energies of $40-200 \mathrm{keV}$ with count rates of up to $140 \mathrm{kcps}$. The detector has an active view on one of the heating beams. The heating beam increases the neutral density locally, thereby, information about the central fast-ion velocity distribution is obtained.

The measured fluxes are modeled with a newly developed module for the 3D MonteCarlo code F90FIDASIM ${ }^{2}$. The modeling allows to distinguish between the active (beam) and passive contributions to the signal. Thereby, the birth profile of the measured fast neutrals can be reconstructed. This model reproduces the measured energy spectra with good accuracy when the passive contribution is taken into account.

\footnotetext{
a) See appendix of ${ }^{3}$
} 


\section{INTRODUCTION}

The diagnosis of supra thermal "fast"-ions is a crucial aspect of high temperature plasma and fusion research. The fast-ion content in the plasma gives directly information about the properties and efficiency of heating and current drive schemes and allows to quantify the interaction of fast-ions with large and small scale plasma instabilities. A comprehensive overview of relevant fast-ion physics is found $\mathrm{in}^{4}$. More recent research makes use of improved diagnostic capabilities to quantify and extend the knowledge of fast-ion physics.

The ASDEX Upgrade (AUG) tokamak is equipped with several fast-ion sources; the neutral beam injection system (NBI) with 8 beams (B1-B8) injects fast particles into the plasma. The ion cyclotron range of frequency (ICRF) heating system utilises radio frequencies to accelerate particles to energies significantly above the thermal level. Therefore, AUG is an ideal testbed for fast-ion studies and allows to test various diagnostic concepts.

The information of distribution and properties of fast-ions is described in fast-ion velocity space coordinates. Due to toroidal symmetry and the strong magnetic field the dimensions, necessary to describe this phase space, are reduced to 4 . The two spatial dimensions $R, z$ span the poloidal plane of the plasma and the two velocity dimensions are the energy of the particle $E$ and its pitch $\xi$, where the pitch is the ratio of the parallel and the total velocity $\xi=v_{\|} / v_{\text {tot }}$.

There are several different diagnostic approaches to obtain information about the fast-ion velocity space, all with their own unique advantages and disadvantages. One is the compact solid state neutral particle analyser (NPA) which is discussed in this paper and whose principle was first tested in $\operatorname{NSTX}^{1}$ and successfully adapted in various experiments ${ }^{5-8}$. At AUG there are also conventional E, B-NPAs ${ }^{9}$, neutron spectroscopy ${ }^{10}$, fast ion loss detectors FILD $^{11}$, collective Thomson scattering CTS $^{12}$ and fast ion d-alpha measurements FIDA ${ }^{2}$.

The compact solid state NPA sacrifices the capability of isotope separation of the conventional NPAs to gain flexibility for the line of sight (LOS) but retains the good energy and pitch resolution. The smaller size reduces the costs for such a system significantly and provides extra flexibility. This made it possible to install the new NPA with a line of sight (LOS) intersecting one of the NBI sources as shown in the toroidal cross-section of AUG in figure 1. Therefore, the new solid state NPA at AUG is called active NPA, while the conventional NPAs with no intersection of LOS and NBI are called passive NPAs. 


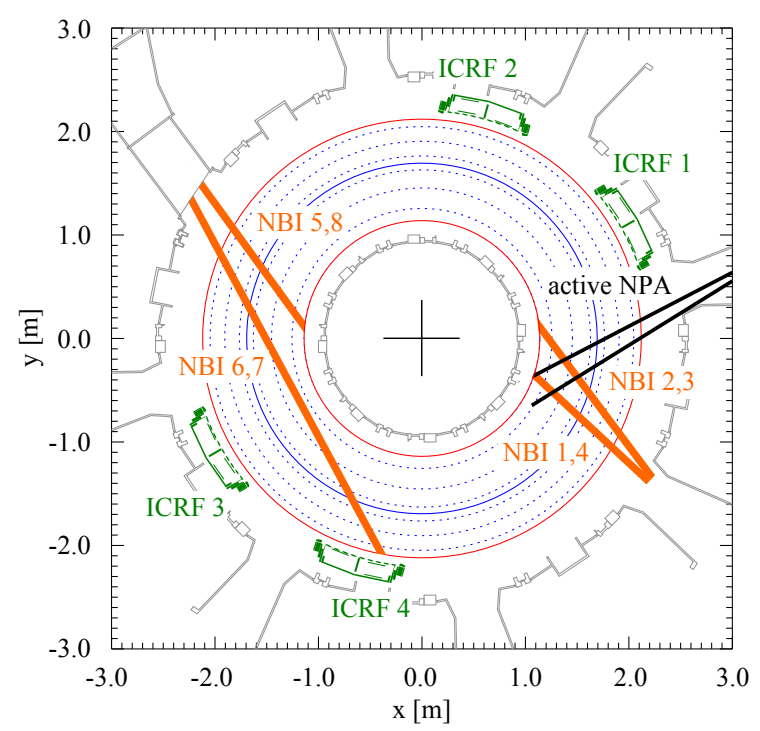

FIG. 1: Top down view of AUG with neutral beam injection geometry, ICRF antennas and two possible line of sights of the active NPA with pitch angles of 0.5 and 0.7 .

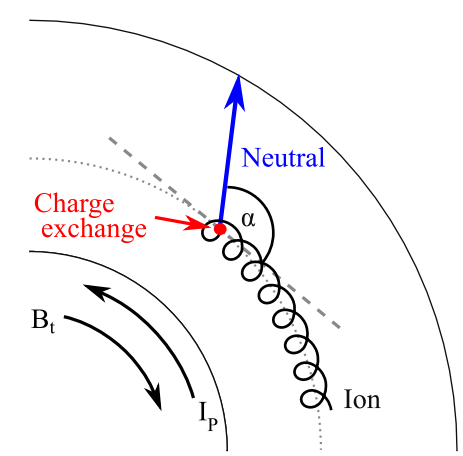

FIG. 2: Sketch of a charge exchange process in AUG.

In section II the basic principle behind the diagnostic is explained and in section III the diagnostic setup is described. In section IV the properties of the new detector at AUG are presented, these include noise and background measurements, the energy calibration and regular measurements of different fast-ion populations. In section $\mathrm{V}$ a synthetic diagnostic based on a Monte-Carlo approach is introduced and compared to measurements. The paper closes with a short summary in section VI.

\section{DIAGNOSTIC PRINCIPLE OF THE ACTIVE NPA}

The NPA diagnostic relies on the charge exchange (cx) of fast-ions with neutral particles in the plasma $\mathrm{D}_{\text {fast }}^{+} \mathrm{D}^{0} \rightarrow \mathrm{D}_{\text {fast }}^{0} \mathrm{D}^{+}$. This process is exploited since the early days of high temperature plasma experiments ${ }^{13}$. A sketch of the charge exchange principle is shown in figure 2. An ion is bound to the strong magnetic field and performs a gyro-motion depending on its pitch. After the charge exchange the neutral still carries the energy and momentum information of the plasma ion, because little energy and momentum are transferred during the charge exchange. The neutral leaves the plasma on a straight line corresponding to its velocity vector at the time of the cx-reaction. The angle $\alpha$ is defined between the new 

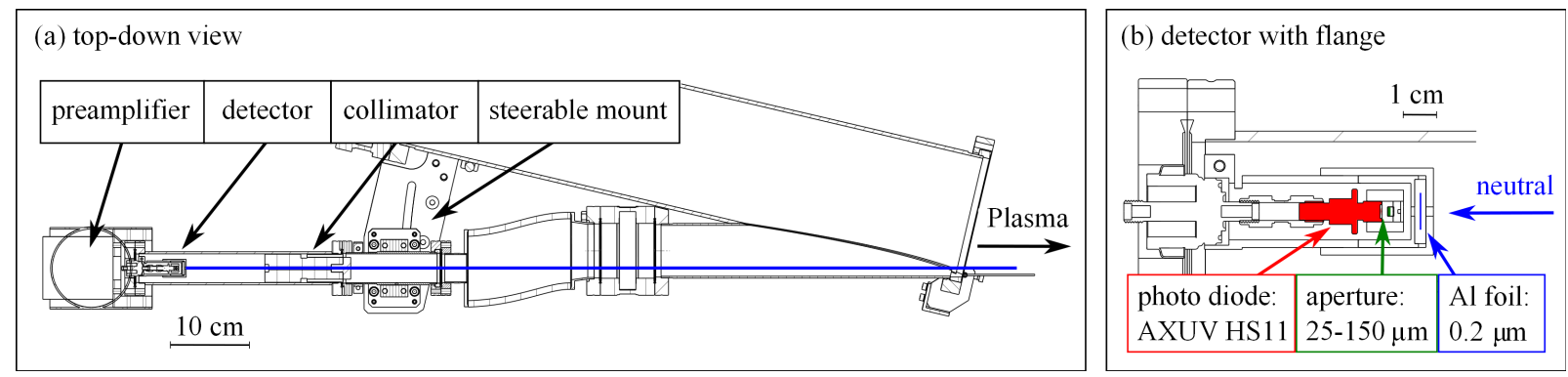

FIG. 3: CAD drawing of the compact solid state NPA at AUG, with a top-down view on

the vacuum tube outside the plasma (a) and a detailed view of the detector head (b).

trajectory and the magnetic field vector, therefore the possible $\alpha$ observed with an NPA is given by the detector's LOS. This angle is correlated with the ratio of the parallel and the perpendicular energy of the ion relative to the magnetic field direction by $v_{\|} / v_{\text {tot }}=-\cos \alpha$. Therefore, the pitch angle observed by the NPA is well defined.

Further information about the fast-ion velocity distribution is contained in the energy and the flux of the neutral particles. The energy of the neutrals is determined with a pulse height analysis of the detector response. The accuracy of this method will be described in section IV B. The reconstruction of the fast-ion density $n_{\mathrm{fi}}$ from the neutral fluxes is not straight forward. The flux of neutrals born in the plasma mainly depends on the convolution of $n_{\mathrm{fi}} \times n_{0}$. Depending on the setup of the system, $n_{0}$ can have two components a passive one from thermal background neutrals $n_{0, \text { pas }}$ and an active component from NBI $n_{0, \text { act }}$. Therefore, one distinguishes between a passive NPA with $n_{0}=n_{0 \text {,pas }}$ and an active NPA with $n_{0}=n_{0, \text { pas }}+n_{0, \text { act }}$. As shown in figure 1 the line of sight used here is an active one. While $n_{0, \text { act }}$ is typically fairly well known ${ }^{14}$, estimates for $n_{0, \text { pas }}$ have large uncertainties. When the birth profile of the fast-ions is known, this does not correspond directly to the measured fluxes, because, neutrals can be re-ionized on their way to the detector. The model used to describe the measured neutral fluxes and their energy spectra with a given fast-ion velocity distribution will be described in section $\mathrm{V}$.

\section{SETUP OF THE ACTIVE NPA}

The NPA is mounted $3.3 \mathrm{~m}$ away from the plasma center at the end of a tube which is connected to one of the AUG A-ports (horizontal ports at the outer midplane) via a bellows. 
This allows to change the measured pitch by tilting the tube in toroidal direction. The tilting is done manually and requires access to the torus hall. Considering the additional influence of the magnetic field curvature this corresponds to an accessible pitch range of $0.5-0.7$. In figure 3 the CAD drawings of the detector is shown outside of the main vacuum vessel (a) and with a zoom onto the detector head (b). The observed solid angle of the diagnostic is determined by a collimator. The detector head consists of an $0.2 \mu \mathrm{m}$ aluminium foil, a variable aperture of $25-150 \mu \mathrm{m}$ and a fast AXUV diode (Opto Diod Corp. AXUVHS11) which is operated at $-50 \mathrm{~V}$ bias. The stainless steel mount for the foil is enclosing the detector, both are connected to the same ground and are electrically isolated from the vacuum tube. The AXUV diode generates a charge pulse $Q$ for each detected neutral with energy $E$, where $Q \propto E$ as will be shown in section IV B. The charge signal is feed through a flange directly to a charge sensitive preamplifier (Canberra 2003BT) and converted into a voltage signal. The distance between detector and preamplifier is less than $5 \mathrm{~cm}$ and the preamplifier is shielded by a copper casing with its own ground. The signal cable as well as the feeder for the preamplifier are also additionally shielded by a conducting mesh. The main amplifier (Canberra Lynx) is set up in a distance of about $10 \mathrm{~m}$ in the so called bunker which provides shielding against neutron and gamma irradiation from the experiment. The main amplifier also acts as a digital signal analyser and uses a pulse height analysis in a time list mode which then yields the time stamp and the energy for each detected event. The information is read out in real time and stored in the AUG discharge database. The the maximal count rate is $140 \mathrm{kcps}$ which is limited by the data acquisition system.

The aperture and aluminum foil thickness is chosen such that the arriving count rate stays below $140 \mathrm{kcps}$. While the aperture reduces the flux for all energies, the aluminum foil blocks low energy neutrals and photons. For deuterium with energies of 30 (100) keV SRIM ${ }^{15}$ simulations yield stopping powers in aluminum of 850 (1500) $\mathrm{MeV} / \mathrm{cm}$. This suggests that particles with energies below $20 \mathrm{keV}$ are shielded effectively by the $0.2 \mu \mathrm{m}$ aluminium foil. Therefore, the energies measured with the detector are between $35-200 \mathrm{keV}$. It is possible to extend the measurement to higher energies by changing the dynamical range of the ADC which will reduce the resolution for lower energies.

The diagnostic is automated; a NPA diagnostic driver prepares the Lynx for data acquisition and stores the data, transmitted by the real time Lynx driver, into the AUG discharge database. The time base is synchronised by an analog TTL trigger pulse distributed at the 


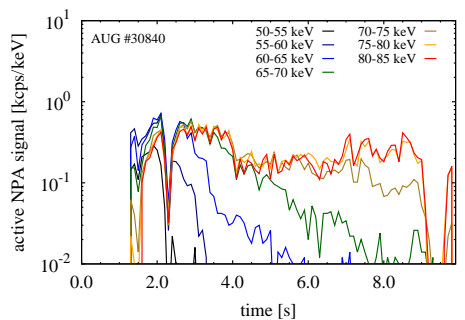

FIG. 4: Count rates of the active NPA measured for different energy intervals during a beam heated AUG discharge.

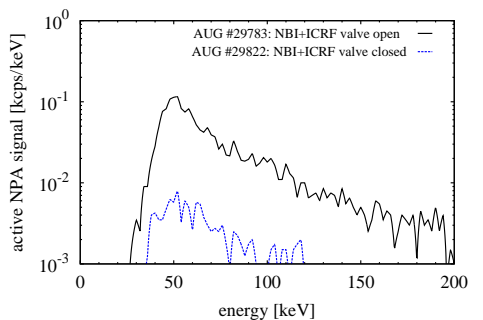

FIG. 5: Measurements of

NPA count rates with open diagnostic valve and neutral particle contribution and closed valve without neutral contribution.

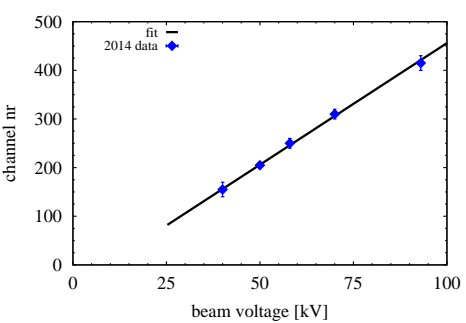

FIG. 6: Digital channel number of measured pulse height vs. beam injection voltage.

beginning of every plasma discharge.

\section{NPA CHARACTERISATION}

\section{A. Background measurements}

Background noise can affect the measured energy spectrum of neutral fluxes in different ways. One can distinguish between two major types; noise on the voltage signal before digitising and noise counts in the energy spectrum after digitising.

The noise on the analog voltage signal can be due to electrical cross-talk, thermal noise or constant photon irradiation. The direct impact on the energy spectrum is expected to be quite small, because, such noise is not falsely interpreted as individual energetic events. In particular, without any fast-particle content in the plasma the count rate, measured by the NPA, is practically zero. This is the case for ECRH heated plasmas with considerable fractions of UV and soft x-ray radiation. However, a large noise on the voltage signal makes it more difficult to discriminate individual events at low energies in the pulse height analysis. This could explain a phenomena observed in AUG high power discharges; over time the count rates for low energies are dropping to zero affecting consecutively higher energies. This is illustrated in figure 4 where the active NPA signal is plotted for different 
energy bins against the time. The signal for $50-55 \mathrm{keV}$ particles is lost already at $2.0 \mathrm{~s}$ while energies of $70-75 \mathrm{keV}$ are only affected for $t>7.0 \mathrm{~s}$. The highest energies $>75$ $\mathrm{keV}$ show the real evolution of the fast-ion content in the plasma which is consistent with FIDA measurements ${ }^{16}$. A possible interpretation is an increasing thermal noise either in the detector or the preamplifier. This is only observed in high power discharges and also more pronounced after multiple high power discharges. However, this hypothesis could not be tested, yet. As a first consequence an active air cooling is installed at the preamplifier for the upcoming experimental campaign.

The main source of noise in spectra are other energetic particles like gammas and neutrons. When interested in the energy spectrum of the main ion species - in most cases deuterium - also fast hydrogen is associated as noise. Because of the very small detector size the number of possible gamma or neutron events is very low and we do not expect realistic spectra. In particular, neutrons do not necessarily trigger a direct detector response, but could also affect the analog voltage noise since the preamplifier is not shielded against neutrons. To quantify this influence two similar discharges with high neutron rates where selected. During one of them the diagnostic vacuum valve was closed. Several mm of stainless steel block all neutral particles and soft x-ray radiation. The measured energy spectra of the gamma/neutron background was found to be one order of magnitude below the signal associated with neutrals as shown in figure 5. Again it is unlikely that electrical cross-talk could produce a similar background spectrum, because, without the energetic particles by NBI or ICRF the NPA measures zero events. However, it cannot be excluded that the ICRF or NBI systems itself are causing such electrical cross-talk.

In AUG a deuterium plasma has always a residual hydrogen content of about $5 \%$. The expected ICRF slowing down distribution for hydrogen minority heating shows a hydrogen population at similar energies as the fast NBI deuterons. However, these contribute little or nothing to the measured spectra of the active NPA. Firstly, for a pitch of 0 the flux ratio of fast hydrogen to deuterium can be measured with conventional passive NPAs and is found to be below $10 \%$. Secondly, the ICRF hydrogen spectra are fairly flat at higher energies and should result in plateus above the beam energy which are not observed in the spectra of the active NPA. 


\section{B. Energy calibration}

The energy calibration of the detector has been performed by analysing the spectra of different discharges with varying neutral beam injection voltages. The detector observes the co-current beam ions directly and does not rely on pitch-angle scattering. Therefore, the full energy component of the injected neutrals is directly visible in the measured energy spectra. With this method an accurate energy calibration is provided for the relevant energy range between $40-100 \mathrm{keV}$. The result of the calibration is shown in figure 6 . The digital channel number of the Lynx, which is proportional to the measured detector response, is plotted against the beam injection voltage. Both are linearly correlated which verifies a linear energy response of the detector. The accuracy of this calibration is about $5 \mathrm{keV}$. At the lowest possible beam injection energy of $30 \mathrm{keV}$ no neutral fluxes were measured. This is explained by the stopping foil in front of the detector and because the pulse height of particles below $10 \mathrm{keV}$ is not distinguishable from the noise on the voltage signal. An extrapolation of the fit towards lower energies does not yield additional information about the detector because of the non-linear energy response due to the stopping foil in this energy range.

Effects not covered with the presented calibration are those due to the diagnostic instrument function. An incident mono energetic particle beam will be broadened. Contributions to the broadening can be scattering in the $0.2 \mu \mathrm{m} \mathrm{Al-foil,} \mathrm{variations} \mathrm{in} \mathrm{the} \mathrm{detector} \mathrm{response}$ and uncertainties in the pulse height analysis of the analogue voltage signal. TRIM simulations of a $100 \mathrm{keV}$ mono-energetic beam propagating through $0.2 \mu \mathrm{m}$ aluminum suggest an energy broadening of the beam by about $5 \mathrm{keV}$. A direct measurement of this broadening in an accelerator facility is planned.

\section{Measurements of different ion populations}

An example for measurements of different fast-ion populations is shown in figure 7 . The plasma discharge exhibits a density ramp (a) which is accompanied by a drop in the electron temperature, thereby, the NBI fast-ion population is changed significantly. Additionally, neutral beams are used to inject ions at different energies, as shown in figure 7 (b). Till 1.0 s only ions with a maximum energy of $59 \mathrm{keV}$ are injected, while two more neutral beam 


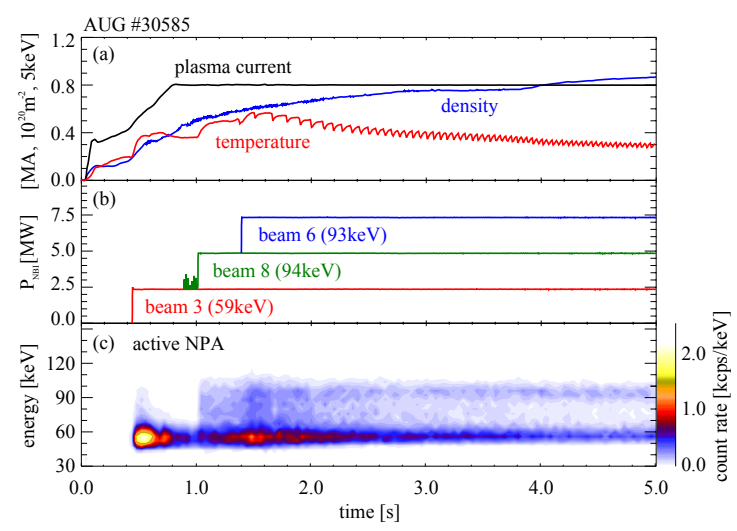

FIG. 7: Time traces plasma current, density and temperature in an AUG discharge (a), different neutral beam heating (b) and a contour plot of the active NPA signal in energy and time $(\mathrm{c})$.

sources are added at 1.0 and $1.4 \mathrm{~s}$. The active NPA signal in (c) measures these changes as expected; at $1.0 \mathrm{~s}$ the measured flux for high energies increases with the $94 \mathrm{keV}$ beam source, with the third source at $1.4 \mathrm{~s}$ the larger fast-ion content is reflected in the increased NPA fluxes for all energies. The impact of the density ramp on the fast-ion slowing down is also nicely observed in the NPA for $t>2.0 \mathrm{~s}$.

This discharge shows only a low noise level on the voltage signal because it was run in the beginning of the day and with relatively low plasma temperatures. Directly with the onset of beam 3 a high energy tail is present which is regularly observed at very low densities, only in the ramp up or ramp down of the plasma, and similar to observations reported for $\mathrm{NSTX}^{1}$. However, no satisfactory explanation for these observation exists yet. In particular, noise due to gammas cannot be excluded for AUG.

\section{SYNTHETIC DIAGNOSTIC}

The measured neutral fluxes give only indirect information about the fast-ion velocity distribution. The birth profile of fast neutrals is a convolution of the $4 \mathrm{D}$ ( $\mathrm{R}, \mathrm{z}, \mathrm{pitch}, \mathrm{energy})$ fast-ion density profile $n_{\mathrm{fi}}$ and the $2 \mathrm{D}(\mathrm{R}, \mathrm{z})$ neutral density profile $n_{0}$ in the plasma. Neutrals born in the plasma have a certain probability to be re-ionised before hitting the detector. This probability depends on their energy and on the background plasma parameters $-T_{\mathrm{e}}$, $n_{\mathrm{e}}, T_{\mathrm{i}}, v_{\text {tor }}, Z_{\mathrm{eff}}$. To distinguish between the influence of the fast-ion density and background plasma parameters on the measured neutral fluxes modelling of the underlying processes is 

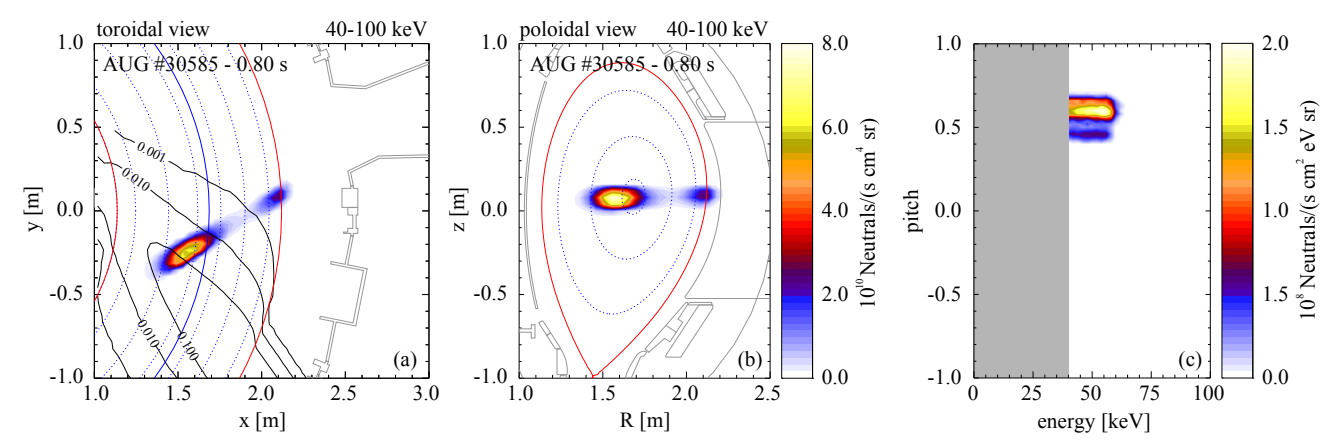

FIG. 8: F90FIDASIM simulation of the birth location of neutrals measured with the active NPA in a toroidal (a) and poloidal (b) projection. The contour lines in (a) illustrate the neutral density due to beam 3 in fractions of its maximum value. Weight function of the simulated neutral fluxes in the velocity space (pitch, energy) (c), energies below $40 \mathrm{keV}$ were not simulated.

required.

The numerical NPA model was implemented as a module in the F90FIDASIM code ${ }^{17}$ which is a further development of the FIDASIM code ${ }^{14}$. F90FIDASIM is a Monte-Carlo code which solves a collisional radiative model for test particles on a uniform 3D grid and simulates the active neutral density due to NBI. It takes into account beam neutrals as well as thermal halo neutrals. The test particles represent fast-ions with densities, energies and velocity vectors depending on a given fast-ion velocity distribution. The velocity distribution is provided to the F90FIDASIM by an independent code, e.g. TORIC ${ }^{18}$ or NUBEAM $(\text { TRANSP })^{19}$. In the NPA mode the spawning of test particles is modified compared to the FIDA mode which reduces the computational time by several orders of magnitude. Only particles are spawned which have a chance to hit the detector, i.e. velocity vector and gyro-angle are pre-selected for each cell. The code calculates the trajectory of the test particles, follows them to solve the collision model in each cell they enter, until they leave the simulation grid. This is done to determine the re-ionisation rate of the neutral particles.

The F90FIDASIM simulation in the NPA mode then yields an energy and pitch resolved $5 \mathrm{D}$ birth profile of the neutral particles. An example is shown in figure 8 which nicely illustrates how one distinguishes between active and passive components in a toroidal projection (a) and a poloidal projection (b). The localisation in the velocity space is shown in figure 8 (c) and illustrates the pitch defined by the detector LOS. The result of the simulation is quite sensitive to the neutral density. Although the shape of the energy spectra is only 
little affected, the absolute fluxes can vary by several orders of magnitude. Experiments with modulation of the active neutral source showed that for low densities $n_{e}<5 \cdot 10^{19} \mathrm{~m}^{-3}$ the passive contribution to the neutral fluxes is about $1 / 3$. For higher densities the neutral beam attenuation is stronger and the re-ionisation rates higher, therefore, the passive component becomes dominant. Simulated fluxes with and without a given $n_{0, \text { pas }}$ profile are shown in figure 9 (a). The edge neutral density was set to a level as expected from SOLPS simulations $^{20}$ and the difference between both cases is supported by measurements of pressure gauges which suggest an increase of the flux of thermal edge neutrals by a factor of 3 . When taking the passive contribution into account experiment and simulation agree well in shape of the spectra for energies below the beam injection energy, as shown in figure 9 (b). Also similar fluxes for both times at $55 \mathrm{keV}$ can be explained. This is not the case when the passive neutral density is neglected as shown in (c).

Above the beam injection energy differences between simulation and measurement are observed which can have the following reasons; it is possible that pile up events in the pulse height analysis cause higher energies, however, the count rate in this discharge is about $20 \mathrm{kcps}$ and therefore significantly below the rate where pile up events are expected. A broad instrument function could also explain the different slopes. However, for a Gaussian instrument function, a FWHM of $20 \mathrm{keV}$ would be required to match the slope which is significantly above the expected broadening due to scattering in the aluminum foil of $5 \mathrm{keV}$. This would still be inconsistent with the two NBI sources at 59 and $93 \mathrm{keV}$, because only one beam can be explained with the broader instrument function as illustrated in figure 10 . Possible physics reasons for an ion population above the beam injection energy are knock-on collisions between fast-ions ${ }^{21}$ and interaction of fast-ions with the thermal electron and ion background ${ }^{22}$. Therefore, an insufficient treatment of these phenomena in TRANSP is also a possible explanation for the observed deviations between simulation and measurement. An independent measurement to exclude instrumental effects is not possible at AUG because the energy resolution of other fast-ion diagnostics like the passive NPAs or FIDA is not high enough. 

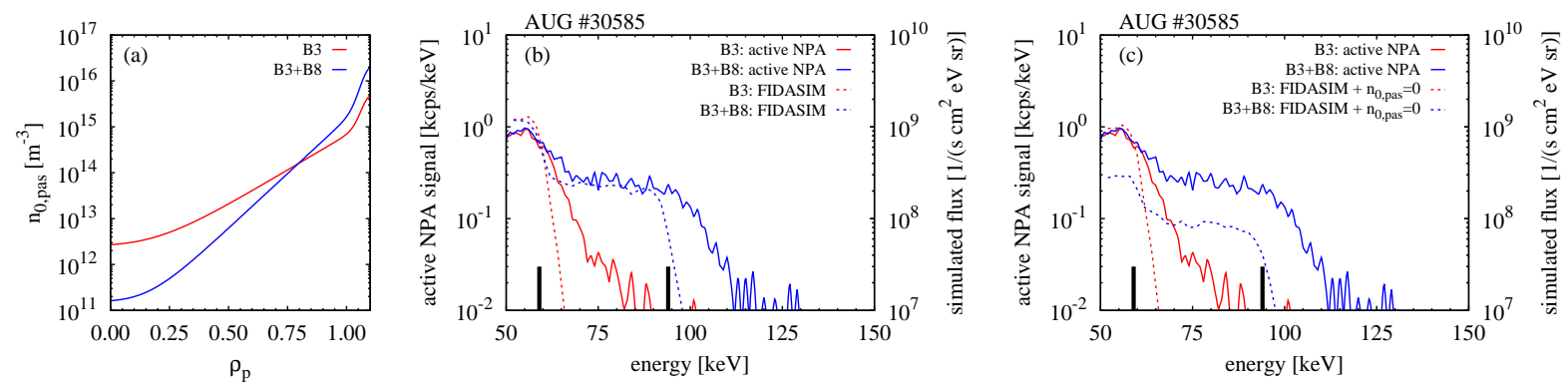

FIG. 9: Neutral density used for the F90FIDASIM simulations with one and two beams (a), the measured active NPA signal compared to the simulations including $n_{0, \text { pas }}$ (b) and the same comparison with only the active component of the neutral flux (c). The beam injection energies are marked in black.

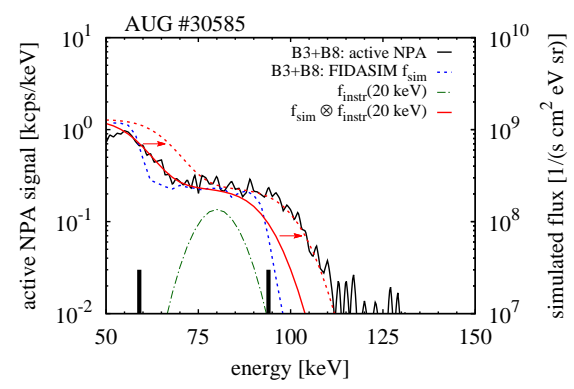

FIG. 10: Energy spectra of the active NPA signal (black) compared to a FIDASIM simulation (blue) and the convolution (red) of the same simulation with a hypothetical instrument function with FWHM of $20 \mathrm{keV}$ (green).

\section{SUMMARY}

A new compact solid-state neutral particle analyser was build, integrated and successfully operated at ASDEX Upgrade. The detector consists of a fast AXUV diode and the incoming neutral rates are determined with a digital signal analyser which uses a pulse height technique to classify individual events. Due to the buffer size and read out bandwidth the possible count rate is limited to $140 \mathrm{kcps}$. To stay below this rate appropriate filter and aperture are used. The detector measures neutral particles above energies of $40 \mathrm{keV}$ and has a good signal to noise ratio of above 10 . Where a noise level of $10 \%$ is only reached in discharges with high neutron rates. The detector can be tilted in between discharges which results in an accessible pitch range of $0.5-0.7$. 
The NPA has an active view on one of the AUG heating beams which allows to obtain information about the central fast-ion velocity distribution. For the interpretation of the measurements an NPA model was implemented in the Monte-Carlo code F90FIDASIM. The model reproduces the energy spectra observed in the measurement with good accuracy when the passive contribution is taken into account. The absolute fluxes have rather large uncertainties due to the influence of fairly unknown passive neutral density. Therefore, the best measurements are obtained at low plasma densities where the active beam neutrals dominate or when beam modulation techniques are applied to directly measure the passive component to the signal.

\section{ACKNOWLEDGMENTS}

This work has been carried out within the framework of the EUROfusion Consortium and has received funding from the European Union's Horizon 2020 research and innovation programme under grant agreement number 633053. The views and opinions expressed herein do not necessarily reflect those of the European Commission.

\section{REFERENCES}

${ }^{1}$ K. Shinohara, D. S. Darrow, A. L. Roquemore, S. S. Medley, and F. E. Cecil, "Solid state neutral particle analyzer array on National Spherical Torus Experiment," Review of Scientific Instruments, 75, 3640 (2004).

${ }^{2}$ B. Geiger, M. Garcia-Munoz, W. W. Heidbrink, R. M. McDermott, G. Tardini, R. Dux, R. Fischer, V. Igochine, and the ASDEX Upgrade Team, "Fast-ion D-alpha measurements at ASDEX Upgrade," Plasma Physics and Controlled Fusion, 53, 65010 (2011).

${ }^{3} \mathrm{H}$ Zohm and E. Al, "Recent ASDEX Upgrade research in support of ITER and DEMO," Nuclear Fusion, 55, 104010 (2015).

${ }^{4}$ W. W. Heidbrink and G. J. Sadler, "The behaviour of fast ions in tokamak experiments," Nuclear Fusion, 34, 535 (1994).

${ }^{5}$ D. Liu, W. W. Heidbrink, D. S. Darrow, A. L. Roquemore, S. S. Medley, and K. Shinohara, "Performance of the solid state neutral particle analyzer array on the national spherical torus experiment," Review of Scientific Instruments, 77, 10F113 (2006). 
${ }^{6}$ V. Tang, J. Liptac, R. R. Parker, P. T. Bonoli, C. L. Fiore, R. S. Granetz, J. H. Irby, Y. Lin, S. J. Wukitch, T. A. C.-M. Team, J. A. Frenje, R. Leiter, S. Mcduffee, and R. D. Petrasso, "Compact multichannel neutral particle analyzer for measurement of energetic chargeexchanged neutrals in Alcator C-Mod," Review of Scientific Instruments, 77, (2006).

${ }^{7}$ Y. B. Zhu, A. Bortolon, W. W. Heidbrink, S. L. Celle, and A. L. Roquemore, "Compact solid-state neutral particle analyzer in current mode," Review of Scientific Instruments, 83, $10 \mathrm{D} 304$ (2012).

${ }^{8}$ D. Liu, W. W. Heidbrink, K. Tritz, Y. B. Zhu, A. L. Roquemore, and S. S. Medley, "Design of solid state neutral particle analyzer array for National Spherical Torus ExperimentUpgradea)," Review of Scientific Instruments, 85, (2014).

${ }^{9}$ R. Bartiromo, G. Bracco, M. Brusati, G. Grosso, S. Mantovani, B. Tilia, and V. Zanza, "Design and calibration of the JET neutral particle analyzer," Review of Scientific Instruments, 58, 788 (1987).

${ }^{10}$ G. Tardini, A. Zimbal, B. Esposito, F. Gagnon-Moisan, D. Marocco, R. Neu, H. Schuhmacher, and the ASDEX Upgrade Team, "First neutron spectrometry measurements in the ASDEX Upgrade tokamak," Journal of Instrumentation, 7, C03004 (2012).

${ }^{11}$ M. García-Muñoz, H.-U. Fahrbach, H. Zohm, and the ASDEX Upgrade Team, "Scintillator based detector for fast-ion losses induced by magnetohydrodynamic instabilities in the ASDEX upgrade tokamak," Review of Scientific Instruments, 80, (2009).

${ }^{12}$ H. Bindslev, J. A. Hoekzema, J. Egedal, J. A. Fessey, T. P. Hughes, and J. S. Machuzak, "Fast-Ion Velocity Distributions in JET Measured by Collective Thomson Scattering," Phys. Rev. Lett., 83, 3206 (1999).

${ }^{13}$ V. V. Afrosimov, P. I. Gladkovskii, S. Y. U. Gordeev, F. I. Kalinkevich, and V. N. Fedovento, "Method of investigation of the flux of atoms emitted by a plasma," Soviet Physics - Technical Physics, 5, 1378 (1961).

${ }^{14}$ W. W. Heidbrink, D. Liu, Y. Luo, E. Ruskov, and B. Geiger, "A Code that Simulates Fast-Ion D_alpha and Neutral Particle Measurements," Communications in Computational Physics, 10, 716 (2011).

${ }^{15} \mathrm{Http}: / /$ www.srim.org.

${ }^{16}$ B. Geiger, M. Weiland, A. Mlynek, M. Reich, A. Bock, M. Dunne, R. Dux, E. Fable, R. Fischer, M. Garcia-Munoz, J. Hobirk, C. Hopf, S. Nielsen, T. Odstrcil, C. Rapson, D. Rittich, F. Ryter, M. Salewski, P. A. Schneider, G. Tardini, and M. Willensdorfer, "Quantification 
of the impact of large and small-scale instabilities on the fast-ion confinement in ASDEX Upgrade," Plasma Physics and Controlled Fusion, 57, 14018 (2015).

${ }^{17}$ B. Geiger, Fast-ion transport studies using FIDA spectroscopy at the ASDEX Upgrade tokamak, Ph.D. thesis, Ludwig-Maximilians-Universität München, München (2012).

${ }^{18}$ R. Bilato, M. Brambilla, O. Maj, L. D. Horton, C. F. Maggi, and J. Stober, "Simulations of combined neutral beam injection and ion cyclotron heating with the TORIC-SSFPQL package," Nuclear Fusion, 51, 103034 (2011).

${ }^{19}$ A. Pankin, D. McCune, R. Andre, G. Bateman, and A. Kritz, "The tokamak Monte Carlo fast ion module $\{$ NUBEAM $\}$ in the National Transport Code Collaboration library," Computer Physics Communications, 159, 157 (2004), ISSN 0010-4655.

${ }^{20}$ F. Reimold, M. Wischmeier, M. Bernert, S. Potzel, D. Coster, X. Bonnin, D. Reiter, G. Meisl, A. Kallenbach, L. Aho-Mantila, and U. Stroth, "Experimental studies and modeling of complete H-mode divertor detachment in $\{$ ASDEX $\}$ Upgrade," Journal of Nuclear Materials, (2014), ISSN 0022-3115.

${ }^{21}$ P. Helander, M. Lisak, and D. D. Ryutov, "Formation of hot ion populations in fusion plasmas by close collisions with fast particles," Plasma Physics and Controlled Fusion, 35, 363 (1993).

${ }^{22}$ C. L. Fiore, S. S. Medley, G. W. Hammett, R. Kaita, A. L. Roquemore, and S. D. Scott, "Ion temperature from tangential charge exchange neutral analysis on the Tokamak Fusion Test Reactor," Nuclear Fusion, 28, 1315 (1988). 